\title{
INTEGRINS EXPRESSED WITH BLADDER EXTRACELLULAR MATRIX AFTER STRETCH INJURY IN VIVO MEDIATE BLADDER SMOOTH MUSCLE CELL GROWTH IN VITRO
}

\author{
JYOTI UPADHYAY, KAREN J. AITKEN, CHRISTOPHER DAMDAR, STEPHANE BOLDUC \\ AND DARIUS J. BÄGLI
}

From the Infection, Immunity, Injury and Repair Division, Hospital for Sick Children Research Institute and Division of Urology, University of Toronto, Toronto, Ontario, Canada

\section{ABSTRACT}

Purpose: It is unknown how bladder smooth muscle cells sense extrinsic mechanical stimuli. The integrins are a large versatile family of transmembrane mechanoreceptors that transduce extracellular matrix (ECM) alterations into the cell, thereby, regulating proliferation, differentiation and ECM synthesis. To our knowledge we provide the first evidence that the integrins may be involved in responses to whole bladder distention and bladder smooth muscle cell stretch.

Materials and Methods: Bladders from 100 to $120 \mathrm{gm}$. rats were stretched to $40 \mathrm{~cm}$. $\mathrm{H}_{2} \mathrm{O}$ for 5 minutes. Five to 96 hours after distention whole bladder mRNAs were isolated for analysis of temporal expression of collagen and integrin genes. Separately quiescent primary culture bladder smooth muscle cells from 1-day-old Sprague-Dawley rats were stretched cyclically for 4 hours. Relative expression of select integrin subunit mRNAs was assessed by semiquantitative reverse transcriptase-polymerase chain reaction. Integrin blockade with asparagine-glycinearginine peptides was used to determine the role of integrins in stretch induced proliferation and the cell cycle in bladder smooth muscle cells.

Results: Within 24 hours bladder distention stimulated collagen expression 2-fold (type I) and 5 -fold (type III). Collagen levels beyond 24 hours were 8-fold (type I) and 2-fold (type III) greater than in controls, revealing an inverse temporal type I-to-III ratio beyond 24 hours. Coordinate alterations were observed in integrin and collagen expression. In vitro bladder smooth muscle cell integrin $\beta 1, \beta 3$ and $\alpha \mathrm{v}$ subunit expression was increased by mechanical stretch $2.5,3.8$ and 5 -fold, respectively, while $\alpha 1$ expression decreased. Asparagine-glycine-arginine peptide inhibition of integrin function significantly inhibited stretch induced bladder smooth muscle cell proliferation and exit from the G2/M phase of the cell cycle.

Conclusions: To our knowledge these results demonstrate for the first time that that bladder distention initiates dynamic alterations in ECM expression. The ability of integrin blockade to suppress stretch induced bladder smooth muscle cell proliferation and the coordinate changes in bladder ECM and integrin expression suggest that integrins mediate key responses to mechanical stimuli in the bladder. Furthermore, cell cycle analysis of resting and stretched bladder smooth muscle cells revealed novel avenues for the examination of integrin and stretch regulation of bladder smooth muscle cell growth.

KEY WoRDs: bladder; muscle, smooth; rats, Sprague-Dawley; extracellular matrix; integrins

To our knowledge the mechanisms regulating bladder smooth muscle cell responses to mechanical stimuli are unknown. Clinically short circuiting prolonged bladder distention by intermittent catheterization is a urological axiom for managing bladders damaged by obstructive uropathy, neurological impairment or voiding abnormalities. ${ }^{1}$ This practice underscores the belief that excessive stretch or prolonged distention are key factors driving recognized fibroproliferative injury to the bladder wall. Experimental distention or partial obstruction of the intact animal bladder ${ }^{2}$ and mechanical stretching of bladder smooth muscle cells ${ }^{3}$ have been shown to produce qualitatively comparable biological responses. However, it is unknown how bladder smooth muscle cells sense these mechanical stimuli.

Generally extracellular matrix (ECM) receptor molecules provide the physical link between cell membrane and surrounding structural ECM proteins, such as collagen and fi-

Accepted for publication August 2, 2002.

Supported by the Physicians Services Foundation of Ontario and American Foundation for Urologic Disease. bronectin. During filling bladder smooth muscle cells become stretched because they are physically attached to the ECM. Integrins are ECM receptors that provide a physicochemical link between cells and the ECM and, thereby, a means to sense alterations in the extracellular environment. The integrins are a large, versatile family of transmembrane mechanoreceptors which, with cell adhesion molecules and selectins ${ }^{4}$ transduce micro-alterations in the ECM environment into the cell..$^{5}$ Furthermore, growth factors can stimulate cell responses through integrins by mutual interaction or integrin clustering with growth factor receptors. ${ }^{6}$ In addition, growth factors and ECM molecules, such as endothelin-1, fibronectin, vinculin and retinoic acid, can regulate the transcription of specific integrin subunits. Composed of heterodimers of $\alpha$ and $\beta$ subunits, the integrins establish dynamic associations between cells and ECM proteins, such as collagen, laminin, fibronectin and vitronectin. Indeed, the integrins were named for their perceived function of integrating the cell surface to the cytoskeleton. ${ }^{7}$

Despite extensive study in other cell systems no data exist 
regarding the role of integrins in bladder smooth muscle cell physiology. Given their role as mechanotransducers it is reasonable to speculate that integrins influence the responses of bladder smooth muscle cells to recurrent mechanical stresses that occur during clinical abnormalities involving bladder filling and emptying. To our knowledge we provide the first evidence that integrins may be involved in responses to whole bladder distention and mechanical stretch of bladder smooth muscle cells.

\section{METHODS}

Rat bladder distention. All experiments and cell explantations were performed under a protocol approved by the animal care committee at our institution. Rat bladders were subjected to acute stretch injury by hydrodistention, as previously described. 8 Briefly, female Sprague-Dawley rats weighing 100 to $120 \mathrm{gm}$. were catheterized via the urethra under general inhalational anesthesia using a 20 gauge angiocatheter. A reproducible distention force was delivered to the bladder (wall stretch) for 5 minutes by filling the bladder with sterile saline to $40 \mathrm{~cm}$. water pressure, as determined by a manometer attached to the catheter. Bladders catheterized but not distended served as negative controls. After distention the bladder was drained and the catheter was removed. At proscribed time points after the procedure the animals were re-anesthetized and the bladder was harvested immediately before sacrifice. Harvested bladders were flash frozen into liquid nitrogen for later mRNA analysis.

Mechanical stretch of bladder smooth muscle cells. Normal rat bladder smooth muscle cell primary cultures were derived, as described previously. ${ }^{8}$ Controlled stretching of smooth muscle cells was performed on an especially designed apparatus (Flexcell Corp., Hillsborough, Connecticut). Smooth muscle cells $\left(1 \times 10^{5}\right.$ per well) were allowed to adhere overnight on deformable tissue culture (carboxyl charged) silicone membranes in culture medium. Cells were stretched at a frequency of $0.17 \mathrm{~Hz}$. (2 seconds of stretch, 4 seconds of relaxation for 6 cycles per minute for $20 \%$ elongation) for 4 hours. Control cells were plated in parallel plates but left unstretched. To assess the role of integrin function during mechanical stretch, linear and cyclic asparagine-glycine-arginine (RGD) peptides was added to smooth muscle cell cultures at 30 minutes before the start of the stretch period.

Reverse transcriptase-polymerase chain reaction (RT-PCR). RNA was isolated from snap-frozen bladder by the acid guadinium thiocyanate-phenol-chloroform method. Tissue was crushed under liquid nitrogen and homogenized with a Polytron (Kinematica AG, Lucerne, Switzerland) in Trizol (Life Technologies, Gaithersburg, Maryland). RNA was then precipitated in isopropyl alcohol, washed in ethanol and dissolved in dihexadecylphosphatidylcholine treated water.

RT-PCR was performed, as previously described. ${ }^{9}$ RNA (2 $\mu \mathrm{g}$.$) was transcribed into cDNA using Superscript II (Life$ Technologies). Oligo deoxythymidine(12-18) (0.5 $\mu \mathrm{g}$.) was added to the RNA and incubated at $70 \mathrm{C}$ for 10 minutes. The cDNA was synthesized in a $20 \mu \mathrm{l}$. volume solution containing RNA, 200 units Superscript II (Roche Diagnostics Basel, Switzerland), $5 \mu \mathrm{M}$. deoxynucleoside triphosphates, $0.01 \mathrm{M}$. dithiothreitol and $1 \times$ buffer $(50 \mathrm{mM}$. tris-Cl, pH 8.3, $75 \mathrm{mM}$. $\mathrm{KCl}$ and $3 \mathrm{mM}$. $\mathrm{MgCl}_{2}$ ). This mixture was then incubated at $42 \mathrm{C}$ for 50 minutes to generate $\mathrm{cDNA}$ and the reaction was terminated at $70 \mathrm{C}$ for 15 minutes. The cDNA was PCR amplified with $200 \mu \mathrm{M}$. deoxynucleoside triphosphates, $1.5 \mu \mathrm{M}$. primers for glyceraldehyde-3-phosphate dehydrogenase $(\mathrm{GAPDH})$, and collagen types I and III $^{9}$ or select $\alpha$ and $\beta$ integrin subunits (see table), $1 \times$ PCR buffer (10 mM. tris, $\mathrm{pH}$ 8.4 and $50 \mathrm{mM}$. $\mathrm{KCl}$ ) with $1.5 \mathrm{mM}$. $\mathrm{MgCl}_{2}, 2$ units Taq DNA polymerase (Invitrogen, Carlsbad, Canada) and $2.0 \mu \mathrm{l}$. cDNA, water or standard. Amplification was then performed. After initial denaturation at 95 for 5 minutes 30 to 35 cycles
Integrin primers

\begin{tabular}{|c|c|c|}
\hline Primer & Sequence & Product Size (bp) \\
\hline $\begin{array}{l}\alpha 1: \\
\text { IntegrinF } \\
\text { IntegrinR }\end{array}$ & $\begin{array}{l}\text { 5'-CATCGTCCTGGATGGCTCCA-3' } \\
\text { 5'-GCAGTCTTGGATGACCTGTT-3' }\end{array}$ & 371 \\
\hline $\begin{array}{l}\alpha \mathrm{v}: \\
\text { IntegrinF } \\
\text { IntegrinR }\end{array}$ & $\begin{array}{l}\text { 5'-CACCGCAGGGTGACTTCATC-3' } \\
5^{\prime} \text {-TAAGGCCACTGGAGGTTCAG-3' }\end{array}$ & 460 \\
\hline$\beta 1:$ & & $234 \underset{(\beta 1 \mathrm{D})}{\beta 1 \mathrm{~A}), 315}$ \\
\hline $\begin{array}{l}\text { IntegrinF } \\
\text { IntegrinR }\end{array}$ & $\begin{array}{l}\text { 5'-TTGTGGAGAACTCCAGACTGTCCTA-3' } \\
5^{\prime} \text {-TCATTTTCCCTCATACTTCGGCTT-3' }\end{array}$ & \\
\hline $\begin{array}{l}\text { B3: } \\
\text { IntegrinF } \\
\text { IntegrinR }\end{array}$ & $\begin{array}{l}\text { 5'-GGGGCTGATGACTGAGAAACT-3' } \\
\text { 5'-GGGCTGAGAGGCTGGGCAAAG-3' }\end{array}$ & 462 \\
\hline
\end{tabular}

of denaturing at $95 \mathrm{C}$ for 30 seconds, annealing at $60 \mathrm{C}$ for 30 seconds, extension at $72 \mathrm{C}$ for 1 minute, and then 10 minutes at $72 \mathrm{C}$ were done. To compare the quantity of each amplification $10 \mu \mathrm{l}$. reaction product were electrophoresed on $1.5 \%$ agarose gel. Band intensities of PCR products were measured under ultraviolet light using a GelDoc 1000 (BioRad Laboratories, Hercules, California) digital camera. Expression levels were analyzed using MultiAnalyst Software, version 2.1.2 (Biorad Laboratories) and normalized to GAPDH transcript levels. Observed band intensity measurements fell within a linear relationship to the amount of template DNA.

Cell cycle analysis. Bladder smooth muscle cell cycle analysis was performed using a modification of published methods. ${ }^{10}$ Cells were plated in serum at $1 \times 10^{5}$ cells per well in Flexcell plates (Flexcell Corp.) for stretch experiments and serum starved for 2 days. Stretched cells or unstretched control cells with or without RGD peptides were washed in phosphate buffered saline and treated with trypsinethylenediaminetetraacetic acid to collect cells. Cells were washed in phosphate buffered saline and fixed in $70 \%$ ethanol. The cells were re-suspended in $20 \mu \mathrm{g} . / \mathrm{ml}$. propidium iodide and $1.8 \mu \mathrm{g} . / \mathrm{ml}$. ribonuclease for 30 minutes on ice and analyzed with a Flow Activated Cell Sorter Scan flow cytometer (488 nm.). Approximately 10,000 cells per sample were sorted during analysis.

\section{RESULTS}

Acute bladder distention stimulated changes in integrin expression. To our knowledge functional bladder integrin mRNA expression has not been previously assessed. We examined how integrins are expressed in the context of bladder hyperdistention or stretch injury. As we reported previously, acute rat bladder distention to $40 \mathrm{~cm} . \mathrm{H}_{2} \mathrm{O}$ in vivo produced transient injury characterized by detrusor fracturing and gross hematuria. A single stretch injury for 5 minutes revealed increases in temporal steady state $\beta 1, \beta 3, \alpha 1$ and $\alpha \mathrm{v}$ integrin mRNA expression (fig. 1).

Brief sustained bladder stretch in vivo stimulated dynamic alterations in ECM gene expression. Since a function of integrins is to sense changes in ECM components such as collagen, we speculated whether gross alterations in integrin transcripts stimulated by stretch was accompanied by coordinate alterations in bladder collagen expression. Sustained stretch injury to the bladder resulted in relatively rapid alterations in mRNA levels of structural collagen types I and III. A single bladder hyperdistention stimulus produced sustained increases in steady state mRNA levels of types I and III fibrillar collagen (fig. 2). However, we observed contrasting profiles of types I and III collagen expression. At less than 24 hours the ratio of type I-to-III was less than 1 . However, beyond 24 hours after stretch the ratio was reversed to greater than 1 . The increase in collagen type III gene expression appeared to be more immediate and sustained.

Mechanical stretch altered $m R N A$ expression of integrin subunits in bladder smooth muscle cells. In vivo bladders 

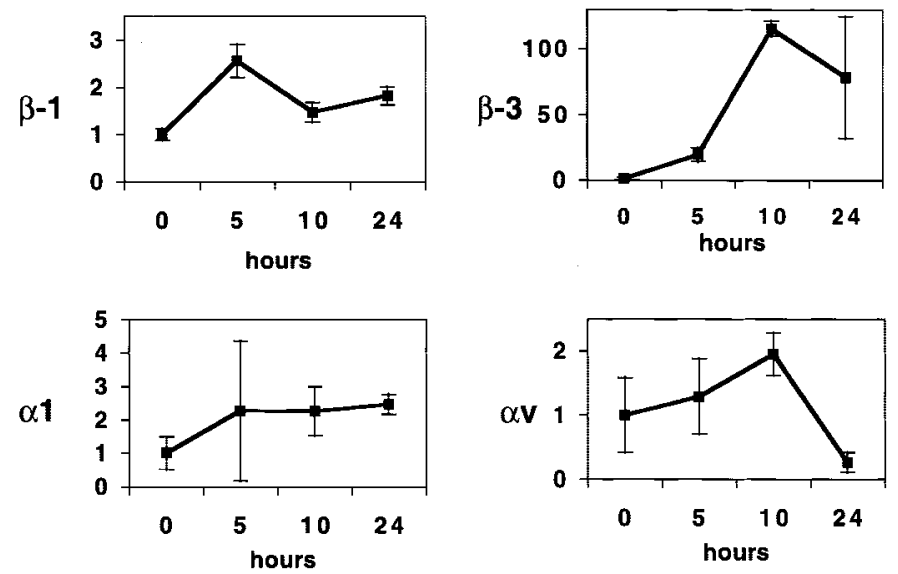

Fig. 1. Expression of integrin subunits in bladders from $120 \mathrm{gm}$. rats hyperdistended in vivo for 5 minutes. After $0,5,10$ and 24 hours $\alpha 1, \alpha \mathrm{v}, \beta 1$ and $\beta 3$ integrin subunit mRNAs were detected by RTPCR. Relative amounts of integrin expression were determined by densitometry comparison with GAPDH mRNA.

represent an intact epithelial-mesenchymal-matrix context in which to study biological responses to stretch. However, this approach does not reveal whether isolated bladder smooth muscle cells show alterations in integrin expression and function in response to mechanical stretching. In this study $\alpha \mathrm{v}, \beta 1$ and $\beta 3$ expression was selectively increased after 4 hours of cyclic mechanical stretch of bladder smooth muscle cells in vitro (fig. 3 ). The expression of $\alpha 1$ decreased after 4 hours of stretch in culture, which may have been related to the minimal increase observed in the whole organ.

Integrins regulated mechanical stretch induced bladder smooth muscle cell proliferation. We next assessed the function of bladder smooth muscle cell integrins in a mechanical stretch context. Bladder smooth muscle cells were stretched in the presence of 2 different RGD peptides. In vitro all cells produce and interact with their secreted ECM proteins, which for bladder smooth muscle cells include collagen and fibronectin. ${ }^{3}$ RGD peptides mimic the binding sites in collagen and fibronectin recognized by many discrete integrins, including those containing $\alpha \mathrm{v}, \beta 1$ or $\beta 3$ subunits. Cyclic and linear RGD peptides interfere with integrin function by competing for binding to cell surface ligands in the ECM. While stretch stimulated bladder smooth muscle cell proliferation in vitro ( $\mathrm{p}=0.005$, fig. 4 ), linear and cyclic RGD significantly inhibited stretch induced proliferation $(\mathrm{p}=0.0001)$. Proliferation in quiescent unstretched cells was also inhibited by linear RGD ( $p=0.0001)$. To our knowledge this observation represents the first evidence that the bladder smooth muscle cell-ECM interaction mediated by integrins is critical to the activation of growth processes during stretch.

The cell cycle is cooperatively regulated by mechanical stretch and integrins in bladder smooth muscle cells. To determine further how integrins may be participating in bladder smooth muscle cell growth we used fluorescence activated cell sorting to analyze the relative number of bladder smooth muscle cells in the various phases of the cell cycle during stretch and/or integrin blockade. Mechanical stretch alone for 4 hours produced an increase in cells entering $\mathrm{G} 2 / \mathrm{M}$ ( $15 \%$ to $20 \%, \mathrm{p}=0.006$, fig. $5, A)$. Cells in the apoptosis, S and G0/G1 phases were not significantly affected by stretching.

In unstretched bladder smooth muscle cells integrin blockade with RGD did not significantly affect the $\mathrm{S}$, G0/G1 or G2/M phase but decreased the number of cells entering apoptosis ( $1.2 \%$ to $0.3 \%, \mathrm{p}=0.04$, fig. $5, A)$. During stretch RGD significantly decreased cells in the G2/M phase (20\% to $17 \%, \mathrm{p}=0.03$, fig. $5, A$ ). Conversely the proportion of cells in the resting phase (G0/G1), repre- senting the majority of all cells at any point, appeared concomitantly increased (fig. 5, B).

\section{DISCUSSION}

Excessive bladder distention and bladder smooth muscle cell stretch are believed to promote maladaptive tissue changes characterized by a fibroproliferative response. While it is universally assumed that bladder smooth muscle cells are stretched during filling, the molecules that provide linkage between them and the surrounding expanding matrix have not been investigated. We explored the role of the integrins, a large and ubiquitous family of transmembrane ECM receptors that mediate cell attachment to the matrix and function as prototypical mechano-transducers. We found that integrins are functionally expressed during brief sustained hyperdistention injury to the intact bladder in vivo. Furthermore, during distention changes in integrin expression and in fibrillar collagen gene expression occur in a coordinate fashion. In addition, cyclic mechanical stretch of bladder smooth muscle cells in vitro stimulates integrin expression and blocking integrin function inhibits stretch induced bladder smooth muscle cell proliferation. Furthermore, mechanical stretch and integrins have discrete effects on the passage of bladder smooth muscle cells through the cell cycle.

For cells to elongate during tissue stretch they must be physically attached to the investing ECM. The integrins are principle molecules for cell attachment to the ECM. ${ }^{7}$ To our knowledge the function of the integrins in bladder smooth muscle cell physiology has not been studied previously. Only a single report describes the immunolocalization of integrin subunits $\alpha 3, \alpha \mathrm{v}, \beta 1$ and $\beta 4$ in the adult human bladder. ${ }^{11}$ No previous assessment of bladder smooth muscle cell integrin function or relationships of bladder distention to matrix expression has been proposed.

We found that bladder hyperdistention modulated the expression of ECM collagen genes. The finding that bladder hyperdistention in vivo produced a type I-to-III collagen mRNA ratio of greater than 1 beyond 24 hours after injury is consistent with a ratio of greater than 1 in the only study to date of this relationship in chronic human fibrogenic bladder. ${ }^{12}$ Our study indicates the first possible mechanistic basis for the ratio greater than 1 observed in the human tissue study.

These observations show that dynamic and potentially relevant alterations in ECM gene expression occur shortly after only a single bladder hyperdistention. Such temporal ECM expression patterns cannot be detected if tissues from fibroproliferative bladder disease are examined only in the established chronic state. Furthermore, these observations may reflect an additional and previously unidentified feature of the regulation of bladder ECM remodeling triggered by distention. The implications of these results are intriguing for understanding the mechanism of clinical disease, in which stretch injuries may be cumulative. Clinically repetitive or cumulative stretch injury may alter collagen gene expression and the matrix sufficiently to create new epitopes or binding sites recognized by integrins. ${ }^{13}$ Our current studies are examining this possibility.

The ubiquitous nature of integrins and their function as canonical mechanoreceptors makes them relevant candidates for study in deciphering the regulation of the bladder smooth muscle cell response to stretch. The precise $\alpha \beta$ heterodimers (intact receptors) operating in the detrusor remain to be determined. It is known that $\alpha \mathrm{v}$ and $\alpha 1$ bind collagen, particularly when it is altered or remodeled, and $\beta 1$ and $\beta 3$ are the principal $\beta$ subunits in $\alpha \beta$ dimers binding collagen and fibronectin. ${ }^{7}$

Integrin expression was responsive to mechanical stretch in the whole bladder context and in primary culture bladder smooth muscle cells. Brief but constant bladder hyperdisten- 

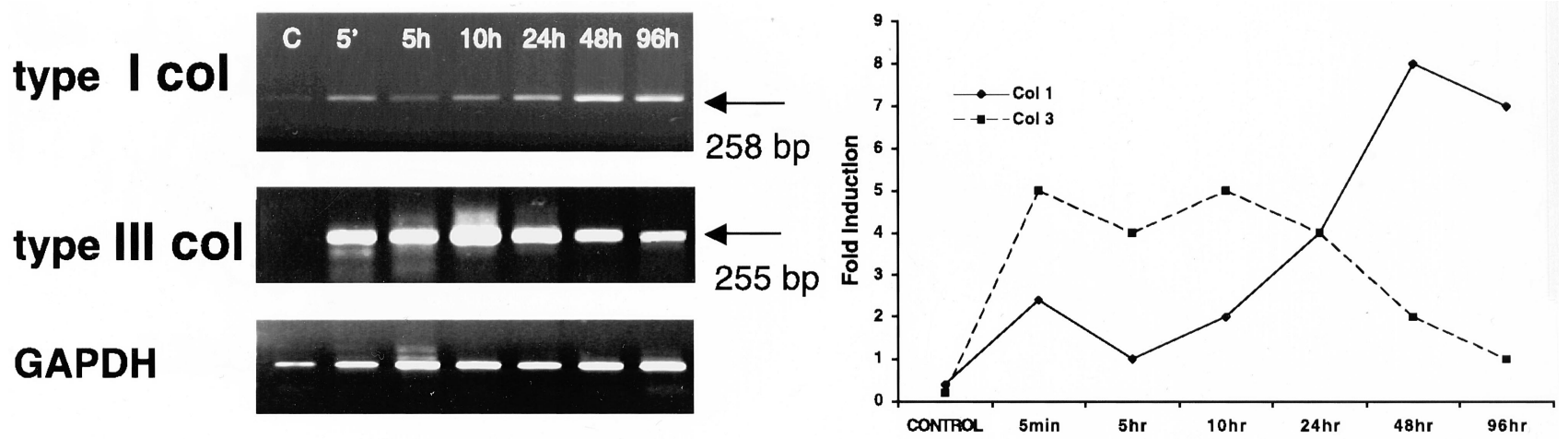

FIG. 2. Hyperdistention regulated collagen gene expression on RT-PCR of types I and III collagen (col) mRNA temporal profiles after 5 -minute sustained hyperdistention in vivo. Note change in types I-to-III collagen ratio from less than 1 to greater than 1 at 24 hours. Northern blot analysis confirmed RT findings (data not shown). Results represent bladders from 2 experiments.
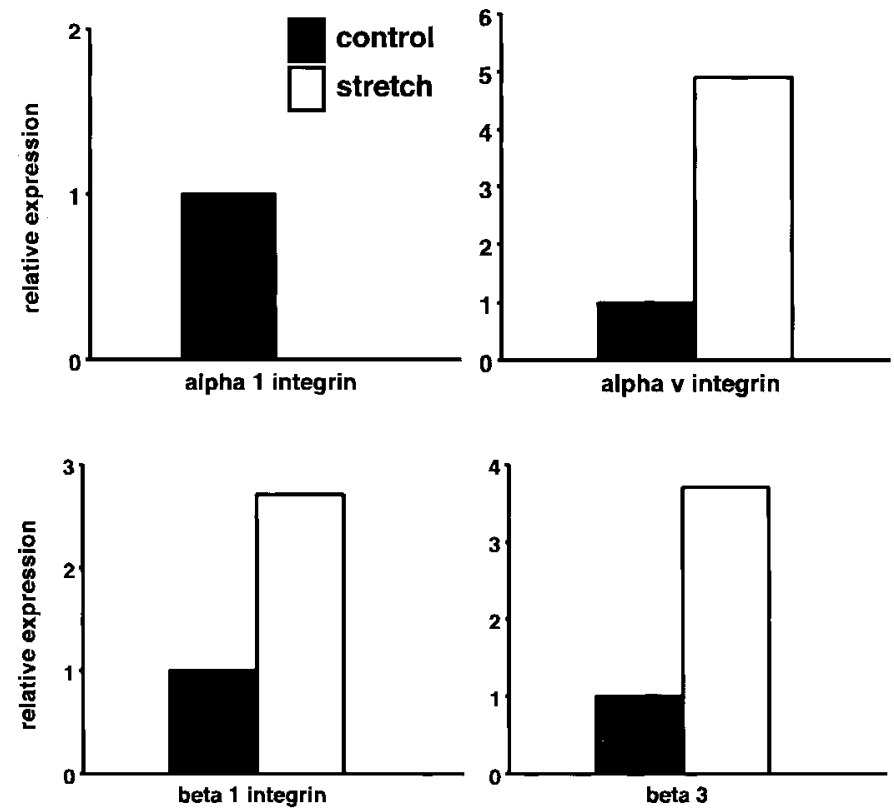

FIG. 3. Stretch induced altered integrin subunit expression in primary neonatal rat bladder smooth muscle cells stretched cyclically with $20 \%$ elongation at $0.17 \mathrm{~Hz}$. for 4 hours. Controls were unstretched bladder smooth muscle cells. Integrin subunit mRNAs were detected by RT-PCR. Relative expression of integrins was determined by densitometry comparison of integrin bands with GAPDH.

tion as well as cyclical bladder smooth muscle cell stretching produced associated alterations in the expression of integrin $\beta 1, \beta 3, \alpha 1$ and $\alpha \mathrm{v}$ subunits. This finding suggests that bladder smooth muscle cell integrins may be stretch responsive in vivo. Whether the changes in integrin expression observed are required for or are a response to the alterations in collagen expression is unknown. While they were similar, integrin expression patterns were not identical in the organ and in cells in 2-dimensional culture. Despite these differences these observations confirm the stretch responsiveness of these ECM integrin receptors in bladder smooth muscle cells. Whether in vivo and in vitro changes in integrin expression correlate physiologically is not yet known since in vitro mechanical stretching is cyclical (stretch-relaxation), in contrast to stretching in vivo, which is sustained (noncyclical) and interrogates integrin expression from all bladder cell types. In other systems a comparison of integrin expression in cultured smooth muscle cells versus tissue revealed discrete alterations in integrin profiles with the highest relative levels of $\alpha 1, \alpha 3, \alpha 5$ and $\alpha \mathrm{v}$ integrins in tissue. ${ }^{14,15}$

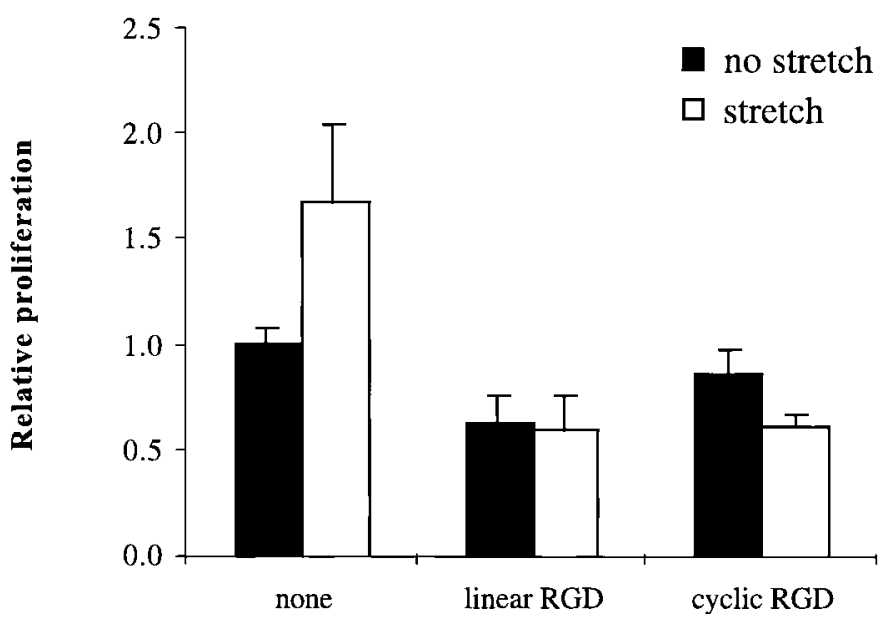

FIG. 4. Integrin function was critical for proliferation of stretched bladder smooth muscle cells, as evaluated by thymidine incorporation into cells stretched $20 \%$ at $0.17 \mathrm{~Hz}$. for 4 hours. Integrin blockade was evaluated by pretreating with linear and cyclic RDG peptides on resting and mechanically stretched bladder smooth muscle cells. Proliferation was significantly higher in stretched than in resting cells $(\mathrm{p}=0.005)$. Pretreatment inhibited stretched stimulated bladder smooth muscle cell proliferation $(\mathrm{p}=0.0001)$

Integrins can provide direct coupling of cells to the matrix and initiate intracellular signal transduction. Thus, an alteration in expression with stretch raises the possibility that subsequent intracellular responses in bladder smooth muscle cell growth and ECM gene expression may be mediated by integrin function. Since bladder smooth muscle cell integrin expression was affected by mechanical stretch, we explored the general role of integrins in bladder smooth muscle cell growth responses. The common peptide motif found in many ECM proteins, RGD, mediates many integrin-ECM interactions, ${ }^{16}$ thus, allowing more than $1 \alpha \beta$ dimer to act as a receptor for a given ligand. This motif and other peptide motifs that mimic it can inhibit integrin ligand binding ${ }^{17}$ and have led to their widespread use in the interrogation of integrin function.

We speculate that $\alpha \mathrm{v}$ and $\beta 3$ integrins may be key ECM ligands regulating stretch induced proliferation in bladder smooth muscle cells. In the current study bladder smooth muscle cell stretch in vitro caused a significant increase in $\alpha \mathrm{v}$ and $\beta 3$ expression. The $\alpha \mathrm{v}$ integrins can recognize the cryptic RGD binding sequences liberated during collagen breakdown. ${ }^{18}$ Expression of $\alpha \mathrm{v}$ integrin confers a prolifer- 

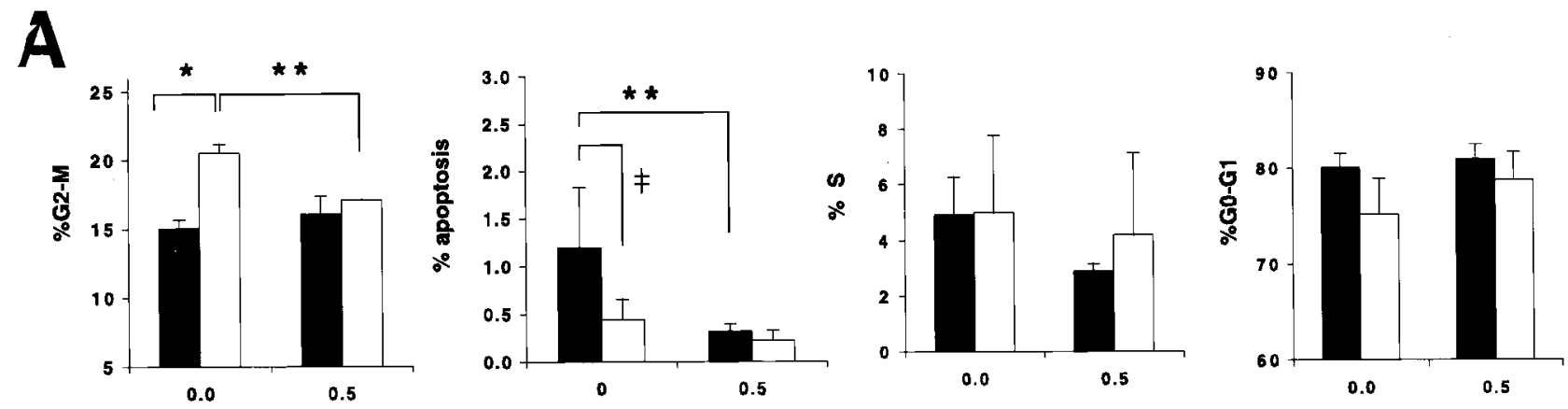

unstretched

stretched

B
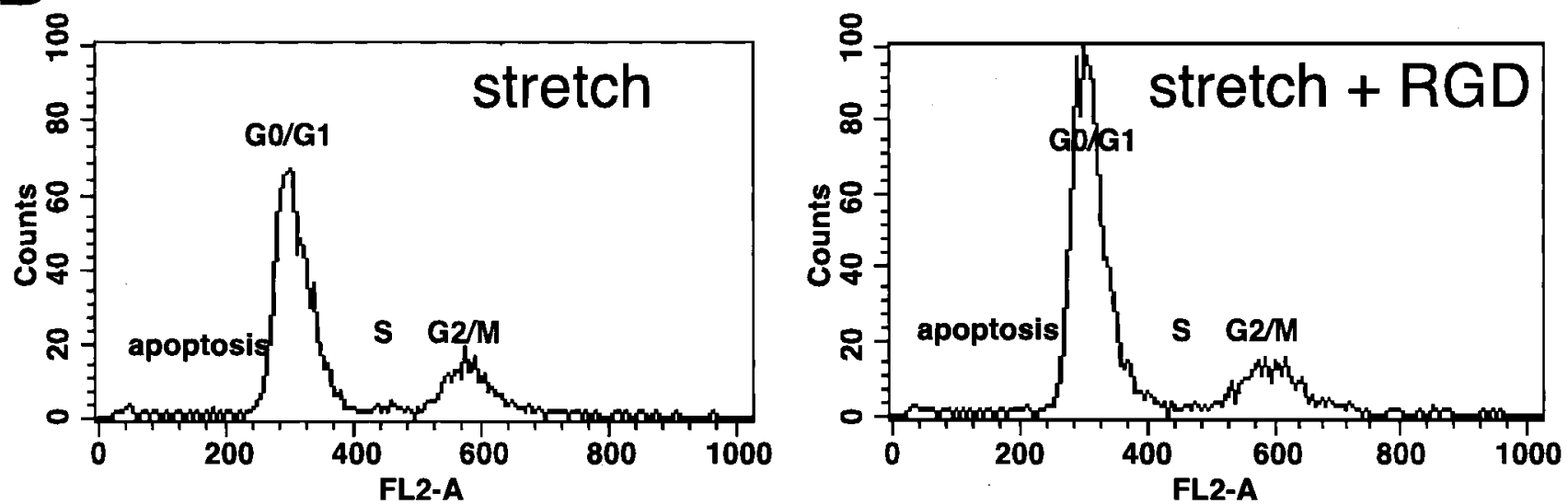

Fig. 5. A, integrin function affected cell cycle progression of primary neonatal rat smooth muscle cells stretched $20 \%$ at $0.17 \mathrm{~Hz}$. for 4 hours. Cell cycle analysis was evaluated by flow cytometry of 10,000 propidium iodide labeled cells per sample in 3 preparations. Effect of cyclic mechanical stretch in presence and absence of linear RGD peptide was compared. Results are expressed as percent of cells per cell cycle phase. Single asterisk indicates $p=0.006$. Double asterisks indicate $p<0.05$. Double dagger indicates $p<0.09$. $B$, area under curve of distribution plot of flow cytometry analysis after RDG treatment of stretched bladder smooth muscle cells represents relative percent of cells per cell cycle phase.

ative advantage in cells cultured on damaged collagen ${ }^{18}$ and smooth muscle cell $\alpha \mathrm{v} \beta 3$ mediated proliferation after vessel injury is blocked by RGD peptide. ${ }^{19}$ Further investigations to delineate the role of $\alpha \mathrm{v} \beta 3$ in stretch injury to bladder smooth muscle cells using mono-specific blocking antibody and antisense oligonucleotides are under way.

We also examined how stretch and integrin function may cooperate in the regulation of bladder smooth muscle cell proliferation using cell cycle analysis. Cells replicate by moving though highly predictable phases of growth (G1 and G2) and synthesis, culminating in their entry into mitosis (M), that is G1 to synthesis to G2/M. In all cells, including the bladder smooth muscle cells studied, the greatest proportion of resting cells always exist in G0/G1. To our knowledge this study represents the first cell cycle analysis of cultured bladder smooth muscle cells. We noted that stretch increased the number of cells entering the G2/M phase and decreased the proportion of apoptosis. A recent report of mechanical stretch in vascular smooth muscle cells suggested that stretch in fact inhibited proliferation by enhancing the number of cells in G0/G1. ${ }^{20}$ While the vascular literature is conflicting in whether mechanical stretch inhibits ${ }^{21}$ or promotes ${ }^{22}$ smooth muscle cell growth, our observations provide new insight into the potential regulatory avenues affecting bladder smooth muscle cell growth in response to stretch since key protein families, such as cyclins and cyclin dependent kinases, as well as growth factors and cytokines are already known to regulate specific phases of the cycle.

The growth inhibitory effects of RGD peptides confirm the notion that integrins participate in key stages of bladder smooth muscle cell growth responses to mechanical stimuli. Integrins appear to have pleitropic effects on the bladder smooth muscle cell cycle. They blunt the entry of stretched cells into G2/M, increase the proportion of cells in the G0/G1 resting phase, and enhance the apoptotic effects of stretch. These observations suggest that key elements of the bladder smooth muscle cell growth response to stretch are potentially regulated by the cyclin dependent kinases, such as cdc2/ cyclin B, that are critical for entry into G2/M. For example, cdc2 expression is increased in dysregulated smooth muscle growth. ${ }^{23}$ In addition, down-regulation of cdk2 by antisense oligonucleotides can prevent vascular smooth muscle cell hyperplasia in response to injury. ${ }^{24}$ We are currently exploring the expression of the relevant cyclins in response to bladder smooth muscle cell stretch and integrin blockade.

\section{CONCLUSIONS}

The increasing appreciation that stretch modulates bladder smooth muscle cell growth and bladder fibroproliferative responses mandates additional studies of the mechanisms 
regulating this process. The long-standing recognition that integrins mediate many cell-matrix responses, including those to mechanical stimulation, ideally positions these transmembrane receptors as candidate molecular mediators of bladder smooth muscle cell growth responses. To our knowledge no functional analysis of integrins in bladder smooth muscle cells has previously been reported.

This study shows that whole bladder distention results in coordinate and dynamic alterations in ECM and integrin gene expression with time. We confirmed that isolated bladder smooth muscle cell cultures also show alterations in integrin expression after mechanical stretch. Furthermore, inhibiting stretch induced bladder smooth muscle cell proliferation with RGD peptides directly implicates integrins in bladder smooth muscle cell growth responses to mechanical stress. Finally in the first analysis of the bladder smooth muscle cell cycle we observed that stretch and integrins cooperate to affect discrete phases of the cell cycle, introducing the possibility that stretch induced bladder smooth muscle cell growth is regulated by critical cdc/cdk proteins activated during bladder smooth muscle cell-matrix interaction. Additional studies of integrin expression and function in the bladder may likely benefit our understanding of stretch mediated bladder disease.

Jenny X. Gu and Christopher McMahon provided technical assistance.

\section{REFERENCES}

1. Weld, K. J., Graney, M. J. and Dmochowski, R. R.: Differences in bladder compliance with time and associations of bladder management with compliance in spinal cord injured patients. J Urol, 163: 1228, 2000

2. Tekgul, S., Yoshino, K., Bagli, D., Carr, M. C., Mitchell, M. E. and Yao, L. Y.: Collagen types I and III localization by in situ hybridization and immunohistochemistry in the partially obstructed young rabbit bladder. J Urol, part 2, 156: 582, 1996

3. Baskin, L., Howard, P. S. and Macarak, E.: Effect of physical forces on bladder smooth muscle and urothelium. J Urol, part 2, 150: 601, 1993

4. Gonzalez-Amaro, R. and Sanchez-Madrid, F.: Cell adhesion molecules: selectins and integrins. Crit Rev Immunol, 19: 389, 1999

5. Wallner, E. I., Yang, Q., Peterson, D. R., Wada, J. and Kanwar, Y. S.: Relevance of extracellular matrix, its receptors, and cell adhesion molecules in mammalian nephrogenesis. Am J Physiol, part 2, 275: F467, 1998

6. Crouch, M. F., Davy, D. A., Willard, F. S. and Berven, L. A.: Insulin induces epidermal growth factor (EGF) receptor clustering and potentiates EGF-stimulated DNA synthesis in swiss 3T3 cells: a mechanism for costimulation in mitogenic synergy. Immunol Cell Biol, 78: 408, 2000

7. Hynes, R. O.: Integrins: versatility, modulation, and signaling in cell adhesion. Cell, 69: 11, 1992

8. Bagli, D. J., Joyner, B. D., Mahoney, S. R. and McCulloch, L.: The hyaluronic acid receptor is induced by stretch injury of rat bladder in vivo and influences smooth muscle cell contraction in vitro. J Urol, 162: 832, 1999

9. Capolicchio, G., Aitken, K. J., Gu, J. X., Reddy, P. and Bagli, D. J.: Extracellular matrix gene responses in a novel ex vivo model of bladder stretch injury. J Urol, part 2, 165: 2235, 2001

10. Horiuchi, A., Nikaido, T., Ya-Li, Z., Ito, K., Orii, A. and Fujii, S.: Heparin inhibits proliferation of myometrial and leiomyomal smooth muscle cells through the induction of alpha-smooth muscle actin, calponin h1 and p27. Mol Hum Reprod, 5: 139, 1999

11. Wilson, C. B., Leopard, J., Cheresh, D. A. and Nakamura, R. M.: Extracellular matrix and integrin composition of the normal bladder wall. World J Urol, suppl., 14: S30, 1996

12. Deveaud, C. M., Macarak, E. J., Kucich, U., Ewalt, D. H., Abrams, W. R. and Howard, P. S.: Molecular analysis of collagens in bladder fibrosis. J Urol, 160: 1518, 1998

13. Herz, D., Aitken, K. and Bagli, D.: Direct stimulation of bladder smooth muscle cell (SMC) growth by altered extracellular matrix (ECM) in vitro is regulated by MAP kinase (MAPK). J Urol, suppl., 165: 86, abstract 352, 2001

14. Belkin, V. M., Belkin, A. M. and Koteliansky, V. E.: Human smooth muscle VLA-1 integrin: purification, substrate specificity, localization in aorta, and expression during development. J Cell Biol, 111: 2159, 1990

15. Mechtersheimer, G., Barth, T., Quentmeier, A. and Moller, P.: Differential expression of beta 1 integrins in nonneoplastic smooth and striated muscle cells and in tumors derived from these cells. Am J Pathol, 144: 1172, 1994

16. Humphries, M. J.: Integrin activation: the link between ligand binding and signal transduction. Curr Opin Cell Biol, 8: 632, 1996

17. Ruoslahti, E.: RGD and other recognition sequences for integrins. Annu Rev Cell Dev Biol, 12: 697, 1996

18. Davis, G. E.: Affinity of integrins for damaged extracellular matrix: alpha $\mathrm{v}$ beta 3 binds to denatured collagen type I through RGD sites. Biochem Biophys Res Commun, 182: 1025, 1992

19. Matsuno, H., Stassen, J. M., Vermylen, J. and Deckmyn, H.: Inhibition of integrin function by a cyclic RGD-containing peptide prevents neointima formation. Circulation, 90: 2203, 1994

20. Chapman, G. B., Durante, W., Hellums, J. D. and Schafer, A. I.: Physiological cyclic stretch causes cell cycle arrest in cultured vascular smooth muscle cells. Am J Physiol Heart Circ Physiol, 278: H748, 2000

21. Dethlefsen, S. M., Shepro, D. and D'Amore, P. A.: Comparison of the effects of mechanical stimulation on venous and arterial smooth muscle cells in vitro. J Vasc Res, 33: 405, 1996

22. Yang, Z., Noll, G. and Luscher, T. F.: Calcium antagonists differently inhibit proliferation of human coronary smooth muscle cells in response to pulsatile stretch and platelet-derived growth factor. Circulation, 88: 832, 1993

23. Zhai, Y. L., Nikaido, T., Shiozawa, T., Orii, A. and Fujii, S. Expression of cyclins and cyclin-dependent kinases in smooth muscle tumors of the uterus. Int J Cancer, 84: 244, 1999

24. Morishita, R., Gibbons, G. H., Ellison, K. E., Nakajima, M., von der Leyen, H., Zhang, L. et al: Intimal hyperplasia after vascular injury is inhibited by antisense cdk 2 kinase oligonucleotides. J Clin Invest, 93: 1458, 1994 